\title{
Finding Four Million Large Random Primes
}

\author{
Ronald L. Rivest* \\ Laboratory for Computer Science \\ Massachusetts Institute of Technology \\ Cambridge, MA 02139
}

A number $n$ is a (base two) pseudoprime if it is composite and satisfies the identity

$$
2^{n-1} \equiv 1(\bmod n)
$$

Every prime satisifies (1), but very few composite numbers are pseudoprimes. If pseudoprimes are very rare, then one could even find large "industrial strength" primes (say for cryptographic use) by simply choosing large random values for $n$ until an $n$ is found that satisfies (1). How rare are pseudoprimes? We performed an experiment that attempts to provide an answer. We also provide some references to the literature for theoretical analyses.

Using a network of 33 SUN Sparcstations, approximately 718 million random 256bit values were tested by a "small divisor test", followed (if the small divisor test was passed) by a test of equation (1), followed (if the equation (1) was satisified) by 8 iterations of the Miller-Rabin probabilistic primality test. A number passes the small divisor test if it has no divisors smaller than $10^{4}$. Of the numbers tested, $43,741,404$ of them passed the small-divisor test. Of those, 4,058,000 satisfied equation (1). Of those, all passed 8 iterations of the Miller-Rabin probabilistic primality test. That is, no pseudoprimes were found. In other words, every number that passed the smalldivisor test and satisfied equation (1) was found to be (probably) prime. Empirically, therefore, pseudoprimes are very rare, at least among numbers with no small divisors.

The available theory also suggests that pseudoprimes are rare. On the basis of extensive experience and analysis, Pomerance $[5,8]$ conjectures that the number of pseudoprimes less than $n$ is at most

$$
n / L(n)^{1+o(1)}
$$

where

$$
L(n)=\exp \left(\frac{\log n \log \log \log n}{\log \log n}\right) .
$$

"Supported by NSF grant CCR-8914428, and RSA Data Security. email address: rivestetheory. Ics.mit. odu

A.J. Menezes and S.A. Vanstone (Eds.): Advances in Cryptology - CRYPTO '90, LNCS 537, pp. 625-626, 1991. (C) Springer-Verlag Berlin Heidelberg 1991 